\title{
High Efficient Link Schedule with Spatial Reuse in Relay Enabled Broadband Wireless Networks
}

\author{
Hanwu Wang,Liping Chen and Tao Yu \\ College of Computer Science and Electronic Engineering, Hunan University, \\ Changsha, Hunan, China
}

Keywords: link bandwidth; link schedule; spatial reuse

\begin{abstract}
The relay enabled broadband wireless access is based on the IEEE 802.16j standard and acts as a promising technology to construct the next generation high speed metropolitan area networks. However, the link schedule and the relevant resource allocation, which is a key challenging issue in such networks, has not been specified in the standard. In this regarding, we propose an efficient link schedule scheme to improve the link traffic scheduling efficiency and resource utilization as well. Specifically we first propose a novel mechanism to exploit the optimal link transmission capacity. Then we design an effective traffic schedule scheme to fully utilize the link transmission bandwidth with respect to user's requirements. Moreover we also propose a spatial reuse scheme to further enhance the link scheduling efficiency and the resource utility efficiency and well. Experiment results validate the efficiency and efficacy of the proposed link schedule schemes.
\end{abstract}

\section{Introduction}

The IEEE 802.16 communication technology provides an attractive wireless access for Internet users. Among all those IEEE 802.16 technologies, the 802.16j[1]defines a new mobile air interface between the base station(BS) and end mobile station(MS). The 802.16 j is based on the 802.16e[2] legacy but extends it to support the mobile multi-hop relay operation by introducing the low cost relay station(RS) into the network. The key purpose of an RS is to aid the good communication between BS and end MSs so that a better transmission speed and range can be achieved. Due to its significant merits, such as high transmission rate, wide coverage, mobility and flexibility, the IEEE 802.16j based relay networks is regarded as a promising alternative for providing broadband wireless access(BWA) in a wide communication area. A general topology for $802.16 \mathrm{j}$ relay network is illustrated in Figure 1.

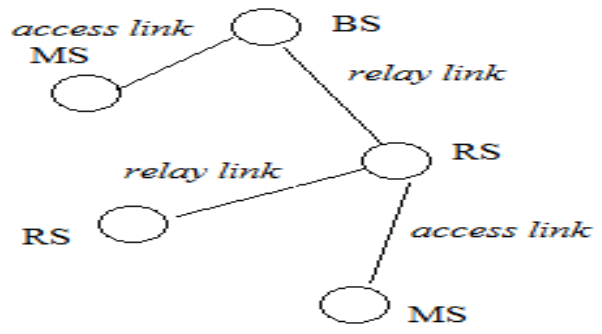

Figure 1 A Simple 802.16j Relay Network Topology

As we can see from figure 1, an 802.16j relay network comprises three types of node, namely BS, RS and MS respectively. Specifically there are two types of links in the network, one is the relay link which is between BS and RS or between two RSs, the other is the access link which is between BS and MS or between RS and MS. Note that the IEEE 802.16j relay network is implemented on a cycle basis and the minimal cycle unit is a so-called frame. In particular each relay frame consists of a downlink subframe and an uplink subframe, which is depicted in Figure 2.

Each subframe is further divided into two time zones, which are named as relay zone and access zone. The relay zone is dedicated to the transmission from BS to RS, or RS to RS, while the access zone is dedicated to the transmission from BS to MS or RS to MS. In this regarding, the MS is the only receiving node in the access zone. 


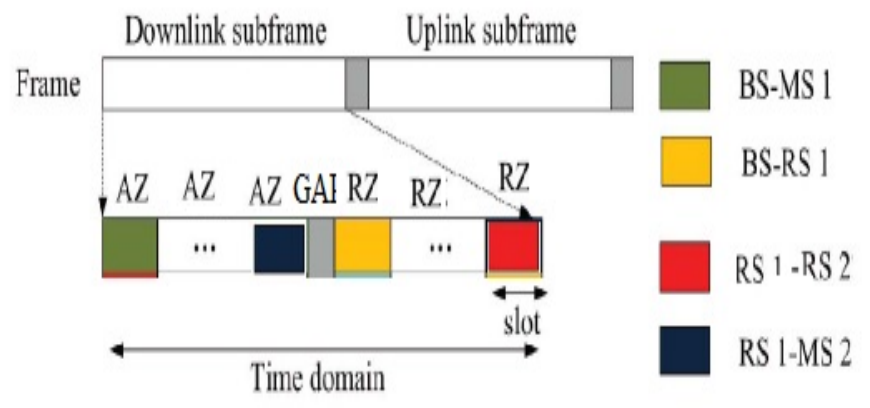

Fig.2A relay network frame structure

In an IEEE 802.16j based relay communication network, the key challenge is how to perform the effective link schedule to meet user individual service requirements. Accordingly, we first propose a link capacity exploiting scheme to maximize the link transmission rate and system capacity. Based on it we further propose an effective link schedule scheme to fully utilize link transmission bandwidth resource with respect to various user requirements.

\section{Link Transmission Capacity Exploiting}

As the wireless link state in a $802.16 \mathrm{j}$ relay communication network may vary from time to time, hence how to adapt the link state variation and exploit the maximal link transmission capacity is a critical challenge. The channel transmission optimization is described as follows. Let $\{[n-1, n)\}$ and $\left\{M L_{\mathrm{n}}\right\}$ be the set of all possible SNR intervals and modulation levels respectively. Suppose an IEEE 802.16j relay frame is composed of $N$ time slots, $\mathrm{t}_{1}, \mathrm{t}_{2}, \ldots, \mathrm{t}_{\mathrm{N}}$, over each time slot $t_{\mathrm{k}}(k=1,2, \ldots, N)$, we first determines its average SNR for an arbitrary link $l$, then we can calculate crossing rate for each SNR level, i.e., $N_{\mathrm{n}}\left(r\left(t_{\mathrm{k}}\right)\right)$. Among all those calculated $N_{\mathrm{n}}\left(r\left(t_{\mathrm{k}}\right)\right)$ values, we choose the maximal one and determine its relevant interval say $\left[n^{*}-1, n^{*}\right)$, and also get the matched modulation level $M L_{\mathrm{n}^{*}}$ and transmission rate $r\left(t_{\mathrm{k}}\right)$ for link $l$. As for the whole frame $\mathrm{f}$, the channel SNR of link $l$ over each time slot $t_{\mathrm{k}}(k=1,2, \ldots, N)$ may vary from one to another, hence one important task is how to partition all those time slots within $f$ into different so called bursts. Specifically a burst denotes a subset of continuous time slots where the channel state of link $l$ over those slots is the same (i.e.,belongs to the same SNR interval). We construct a new burst by adding each appropriate slot into it in a sequence manner. As for each new burst $B_{\mathrm{i}}$, we first determine the desired channel state say $n^{*}$ for its first time slot $t_{s}$, then similarly we work on the next time slot $t_{e}$ and get its SNR state. If two states are the same, then we can add $t_{\mathrm{e}}$ into burst $B_{\mathrm{i}}$ and further handle the remaining time slots; otherwise we let $\mathrm{t}_{\mathrm{e}}$ be the first time slot of another new burst. For each round we can work out one burst with a definite channel state and its matched transmission rate $r\left(l, B_{\mathrm{i}}\right)$. In this way we can readily get all possible transmission bursts within $f$ for link $l$.

\section{Link Schedule on Traffic Flows}

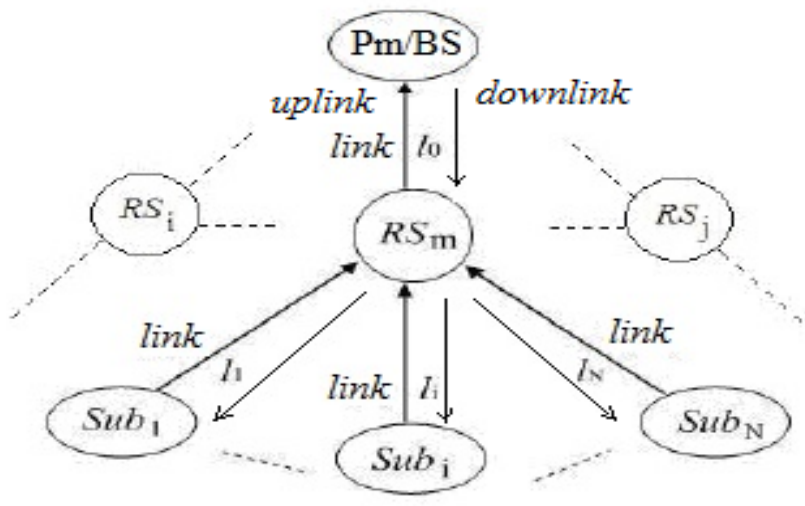

Fig.3. Link Schedule Model 
In this section we further analyze how to utilize the available link bandwidth resource to schedule the network traffic flows to meet the desired performance objectives. Note that traffic flows in 802.16j relay access system can be divided into two classes: one is the QoS flow[3][4] such as UGS, ertPS, rtPS, nrtPS with specific requirement on bandwidth and delay; the other is the Non-QoS flow (i.e., BE) without any QoS requirement. All the BE traffic flows will contend for the available bandwidth in a best effort manner. Here we propose an effective link schedule (ELS) scheme to optimize the link transmission bandwidth resource over the whole networks.

Let's first consider a link schedule model, as illustrated in Figure 3 for analyzing traffic transmission and schedule in IEEE 802.16j relay network, where station $R S_{\mathrm{m}}$ represents a generic relay station (RS) within the network. Stations $S u b_{1}, S u b_{i}, \ldots, S u b_{N}$ are the $N$ subordinate stations of $R S_{\mathrm{m}}$, while $P_{\mathrm{m}}$ is its superordinate station. Links $l_{1}, l_{\mathrm{i}}, \ldots, l_{\mathrm{N}}$ are the relevant subordinate links and link $l_{0}$ is the superordinate link. Suppose the total available resource (i.e., time slots or bursts) allocated to the transmission model (regarding $R S_{\mathrm{m}}$ ) in Fig. 2 is denoted by $A(m)$. Specifically we can define a utility function, say, $U t(A(m))$ among the competing links as

$$
U t(A(m))=\sum_{i=0,1, \ldots, N}\left[\sum_{B_{k} \in a\left(l_{i}\right)} r\left(l_{i}, B_{k}\right) \times\left|B_{k}\right|\right]
$$

where $r\left(l_{\mathrm{i}}, B_{\mathrm{k}}\right)(i=0,1,2, \ldots, N)$ denotes the normalized channel transmission rate over link $l_{\mathrm{i}}$ with respect to burst $B_{\mathrm{k}}$. Parameter $a\left(l_{\mathrm{i}}\right)$ denote the subset of time slots (bursts) allocated to link $l_{\mathrm{i}}$. Hence the utility function in Eq. (3) represents the total transmission throughput produced at the schedule model regarding resource $A(m)$.

Our objective is to maximize the utility of the link transmission bandwidth resource by achieving both the QoS performance satisfaction and optimized system transmission throughput. Accordingly we formulate the following objective optimization problem as

$$
\begin{aligned}
& \text { Maximize } \quad U t(A(m)) \\
& \text { Subject to } \\
& \text { QoS demand on rate } \\
& X\left(f_{i}\right)=R\left(f_{i}\right) \\
& \text { QoS demand on delay } \\
& W\left(f_{i}\right) \leq W_{\max }\left(f_{i}\right)
\end{aligned}
$$

Formulation (2) aims to maximize system transmission throughput with respect to QoS performance constrains of (3), (4). Specifically the transmission rate $X\left(f_{i}\right)$ allocated to a QoS flow $f_{i}$ should meet its rate demand $R\left(f_{i}\right)$ without contention. While the waiting time $W\left(f_{i}\right)$ for QoS flow $f_{i}$ should not exceed its maximal tolerant delay (deadline) $W_{\max }\left(f_{i}\right)$. Due to different performance requirements, we deal with QoS and Non-QoS traffic via different strategies: As for those QoS traffic flows, we handle them in a priority manner so that the one(s) with the most urgent delay demand are chosen for transmission at each schedule round. As for those Non-QoS traffic flows, we handle them in an opportunistic manner to achieve a maximal system traffic throughput.

Aside from the above effective link schedule (ELS) scheme, if we only focus on maximizing the system traffic throughput, then each available resource (burst) will be allocated in a greedy manner among different links, so that the link with the highest transmission rate will be preferred at each schedule round. We call this scheme as the opportunistic link schedule (OLS) scheme, which will be used to make a comparison between the ELS and OLS scheme in the experiment part.

\section{Exploiting Spatial Reuse}

In this section we further study the spatial reuse exploitation to improve the link scheduling efficiency in IEEE 802.16j relay networks. Note that spatial reuse[6][7] is based on the fact that different links can be concurrently schedule provided that they do not interfere with each other. Utilizing spatial reuse consequently can achieve better link transmission efficiency and bandwidth resource efficiency. However how to realize the spatial reuse over the whole relay network is not a trivial issue. 
Specifically we present a working mechanism for spatial reuse exploitation which includes modules of the concurrent link subsets partition, transmission resource assignment, and spatial reuse schedule. We will elaborate them as follows. Firstly we should partition all those transmission links over the whole relay network and get various subsets of concurrent links. A subset of concurrent links represents a group of links which can be activated simultaneously, while different subsets will be scheduled in different time instants. The subsets can be constructed starting from an arbitrary link as follows. Given an arbitrary link $l$, we first set an initial subset for it and then we search all the possible concurrent links for link $l$. For each new link $l$ ', if it does not interfere with any links within the subset of link $l$, we then add link $l$ ' into the subset. Otherwise we discard link $l$ ' and search another one. If all the other links have been handled, we then start with another link and construct the subset for it in a similar way. In this way we can construct all the concurrent subsets over the whole relay network. As for the transmission resource assignment, we should allocate the time slots resource among all those subsets. Note that the time slot assignment for each subset is related to resource demand of the subset. Suppose the resource demand for each subset is $d\left(S_{1}\right), d\left(S_{2}\right), \ldots, d\left(S_{\mathrm{M}}\right)$, then the time slot allocation for these subsets are determined as $a\left(S_{1}\right): a\left(S_{2}\right): \ldots: a\left(S_{\mathrm{M}}\right)=d\left(S_{1}\right): d\left(S_{2}\right): \ldots: d\left(S_{\mathrm{M}}\right)$. As for the spatial reuse schedule, we should schedule different subsets in a sequence manner, while schedule different link within the same subset in a concurrent manner, i.e., those links of the same subset can be simultaneously activated.

\section{Conclusion}

This paper aims to exploit the optimization on radio channel resources in $802.16 \mathrm{~m}$ networks to meet the user application requirements. To conquer the challenges caused by the state variation of communication channels, we designed the novel schemes to specify the channel state and exploit the maximal channel transmission capacity. Moreover, an effective channel usage scheme is proposed to fully utilize the channel bandwidth regarding the user actual demand. We also make the performance analysis with the adoption of network calculus for the proposed schemes.

\section{Acknowledgements}

This work is fully supported by National Natural Science Foundation (NSF) of China with grant No.61202461, and National Natural Science Foundation (NSF) of China with grant No. 61472129.

\section{References}

[1] S.W. Peters and R.W. Heath, The Future of WiMAX: Multihop Relaying with IEEE 802.16j, IEEE Communications Magazine, vol. 47, no. 1, pp. 104-111, 2009.

[2 I. S. 802.16e 2005, IEEE standard for local and metropolitan area networks part 16: Air interface for fixed broadband wireless access system Proceedings of IEEE Standards, 2005.

[3] Jeffrey G. Andrews and Arunabha Ghosh and Rias Muhamed, Fundamentals of WiMAX: understanding broadband wireless networking, Upper Saddle River, NJ: Prentice Hall,2007.

[4] IEEE Standards, IEEE Draft Standard for local and metropolitan area networks Part 16: Air Interface for Broadband Wireless Access Systems, pp. 1-2614, March 2012.

[5] M. -S. Alouini and A. J. Goldsmith, Adaptive modulation over Nakagami fading channels, Kluwer Journal on Wireless Communication, vol. 13, no. 1-2, pp. 119-143, 2000.

[6] R. Krenz, Wireless mesh network capacity analysis with spatial channel reuse and adaptive modulation, International Symposium on Communication Systems, Networks \& Digital Signal Processing (CSNDSP), pp. 1-5, 2012.

[7] M. I. Rafique, Exploiting smart antennas for spatial reuse and multiplexing in wireless mesh networks, IEEE 14th International Symposium and Workshops on a World of Wireless, Mobile and Multimedia Networks(WoWMoM), pp. 1-7, 2013. 